Published in final edited form as:

Psychiatry Res. 2016 December 30; 246: 230-235. doi:10.1016/j.psychres.2016.09.044.

\title{
Problematic sexual behavior in young adults: Associations across clinical, behavioral, and neurocognitive variables
}

\author{
EW Leppink(1), SR Chamberlain ${ }^{(2)}$, SA Redden ${ }^{(1)}$, and JE Grant ${ }^{(1),{ }^{*}}$ \\ ${ }^{1}$ Department of Psychiatry, University of Chicago \\ ${ }^{2}$ Deparment of Psychiatry, University of Cambridge
}

\begin{abstract}
Objective-A notable number of young adults struggle to control impulsive behavior, resulting in impairment and distress. Assessments of problematic sexual behavior (PSB) have noted clinical differences relative to other populations, but neurocognitive findings have varied. This analysis assesses the clinical presentation and neurocognitive profile of patients with PSB relative to participants evident PSB symptoms.
\end{abstract}

Methods-492 participants (18-29) were recruited for a study on impulsivity in young adults. Participants completed diagnostic, self-report, and neurocognitive measures which assessed several cognitive domains. PSB was defined as endorsing fantasies, urges, or sexual behavior that felt out of control or was causing distress.

Results-54 (11\%) participants reported current PSB. This group was older, reported earlier sexual experiences and alcohol use, and lower quality of life and self-esteem. Comorbidity was greater in the PSB group, particularly for depression and alcohol dependence. The PSB group also showed differences in impulsivity, decision making, spatial working memory, problem solving, and emotional dysregulation.

Conclusion-Results suggest that PSB is associated with psychosocial dysfunction, greater comorbidity, and neurocognitive differences. These associations suggest a more salient impact than typical sexual behavior. Furthermore, this study demonstrated several neurocognitive deficits in the PSB group which have found more mixed support previously.

\section{Keywords}

comorbidity; neurocognition; cognition

*corresponding author jgrant4@bsd.uchicago.edu.

Conflicts of interest

Dr. Grant has received research grants from the National Center for Responsible Gaming, the American Foundation for Suicide Prevention, Brainsway, and Forest, Takeda, and Psyadon Pharmaceuticals. He receives yearly compensation from Springer Publishing for acting as Editor-in-Chief of the Journal of Gambling Studies and has received royalties from Oxford University Press, American Psychiatric Publishing, Inc., Norton Press, McGraw Hill, and Johns Hopkins University Press. Dr Chamberlain's involvement in this research was funded by a grant from the Academy of Medical Sciences (UK). Dr Chamberlain consults for Cambridge Cognition. Mr. Leppink and Ms. Redden report no financial relationships with commercial interests. 


\section{Introduction}

Sexual behaviors, including sexual risk-taking and experimentation, are common among young adults (Kaestle et al., 2004; Kann et al., 2014; Santelli et al., 1998). Some individuals, however, have problems controlling their sexual urges and/or behaviors. Young adulthood is also frequently associated with numerous impulsive behaviors in general, including alcohol abuse and illegal drug use (Chen et al., 2005; Courney and Polich, 2009; Kann et al., 2014; Young et al., 2002). In some cases, sexual and other risk-taking behaviors start to reflect a pattern of impulsivity resulting in significant impairment and distress. Although sexual behavior may be fairly common among young adults, it is unclear how many young adults experience problems with sex. Problematic sexual behavior has been relatively understudied across the lifespan, particularly in young adults.

In the present study, we assessed a large sample of non-treatment seeking young adults regarding sexual behaviors. Although previous research suggests that compulsive sexual behavior and other addictive behaviors may be linked, no study has systematically examined the relationship of problematic sexual behavior to a range of behaviors and cognitions (Black et al., 1997; Derbyshire and Grant, 2015; Kuzma and Black, 2008). For purposes of this study, we chose to examine sexual behaviors reflective of an unhealthy or problematic level (characterized by a combination of repetitive sexual fantasies, urges, or behavior that is perceived to be out of control or cause significant distress) without over-pathologizing the behavior as a psychiatric disorder (as could be the case in hypersexuality or compulsive sexual behavior). A similar approach has been used with other problematic behaviors, such as hazardous drinking and higher risk gambling, in order to assess the impact of these behaviors on clinical presentation and functioning (Agrawal et al., 2010; Carneiro et al., 2014). We hypothesized that PSB would be reported frequently, would be associated with a range of impulsive behaviors, and would be associated with underlying cognitive dysfunction relative to young adults with no history of PSB. Examining a problematic level of sexual behavior, that does not reach diagnostic criteria for a sexual disorder, may have important public health implications, particularly for early interventions and education.

Given the incomplete data on problematic sexual behavior among young people, particularly in community samples, the aims of this study were to: 1) examine the prevalence and sociodemographic correlates of problematic sexual behavior in young adults; 2) investigate mental health correlates in young adults who report problematic sexual behavior; and 3) examine the neurocognitive underpinnings in young adults with sexual thoughts/behaviors indicative of this problem.

\section{Methods}

A sample of 491 participants was recruited from the surrounding community near two large Midwestern universities for a study on impulsive behavior in young adults. PSB was assessed using the Minnesota Impulsive Disorders Interview (MIDI) (Odlaug and Grant, 2010) and was defined as a response of "Yes" to any of the 4 primary diagnostic questions from compulsive sexual behavior module, listed below: 
1. Do you or others that you know think that you have a problem with being overly preoccupied with some aspect of your sexuality or being overly sexually active?

2. Do you have repetitive sexual fantasies which you feel are out of your control or cause you distress?

3. Do you have repetitive sexual urges which you feel are out of your control or cause you distress?

4. Do you engage in repetitive sexual behavior which you feel is out of control or cause or distress?

All participants also completed standard diagnostic interviews, basic demographic information, self-report impulsivity inventories, and a computerized cognitive battery. Psychiatric comorbidity was assessed using the Mini International Neuropsychiatric Inventory (MINI) (Sheehan et al., 1998) by trained raters. All study procedures were carried out in accordance with the Declaration of Helsinki. The Institutional Review Boards of the University of Minnesota and of the University of Chicago approved the procedures and the accompanying consent forms. All participants provided written informed consent prior to participation in the study.

\subsection{Clinical measures}

Minnesota Impulsive Disorders Interview (MIDI) (Odlaug and Grant, 2010): The MIDI is a self-report inventory which screens for several impulse control disorders including the following: CSB, kleptomania, intermittent explosive disorder, gambling disorder, compulsive buying, skin picking disorder, trichotillomania, pyromania, and binge eating disorder. Where available, the MIDI uses criteria set by the DSM- 5 to identify individual disorders, including skin picking, trichotillomania, gambling disorder, and binge eating disorder. The MIDI has been used previously to assess the prevalence of impulse control disorders in several samples with good reliability (Odlaug and Grant, 2010).

\subsection{Self-report measures}

Barratt Impulsiveness Scale, Version 11 (BIS) (Barrett, 1959; Patton et al., 1995): The BIS is a self-report measure of impulsivity across attentional, motor, and non-planning dimensions. The measure consists of 30 questions, with each rated on a scale of 1 ("Rarely/Never") to 4 ("Almost Always/Always"). Second-order scores are reported for the dimensions of attentional, motor, and non-planning impulsivity.

Rosenberg Self-Esteem Scale (RSE) (Rosenberg, 1965): The RSE is a 10 question selfreport inventory which assesses levels of self-esteem. Factors assessed include feelings of satisfaction with oneself, worth, and attitude towards oneself amongst others. Responses range from "Strongly Disagree" to "Strongly Agree", and yield a composite score.

Difficulties in Emotion Regulation Scale (DERS) (Gratz and Roemer, 2004): The DERS is a self-report measure of emotional dysregulation. The measure consists of 36 questions with responses ranging from 1 ("Almost Never") to 5 (“Almost Always"). The target aspect of the measure for this analysis was the composite score of the scale. 
Quality of Life Inventory (QOLI) (Frisch et al., 1992): The QOLI is a 32 question self-report measure of perceived quality of life. Participants are asked to provide answers of how important a given factor is on a scale from 0-2, and then an answer of how satisfied they are with that factor on a scale of $-3-3$. These values are then multiplied to give a net score for that factor. Factors are then summed to give a raw score. Scores are then converted into tscores for the final analysis using the methods reported by Frisch and colleagues (Frisch et al., 1992).

\subsection{Cognitive measures}

Neurocognitive variables were assessed using the Cambridge Neuropsychological Test Automated Battery (CANTAB) system. The following assessments were included in this analysis:

Intra-/Extra-dimensional Set Shift (IDED): The IDED assesses cognitive flexibility, which is associated with compulsivity. During the task, participants are presented with four boxes, two of contain pink shapes. Participants are told that one shape has been chosen as "correct", and the remaining is "incorrect". They are then informed that their goal is to select the correct shape as many times as possible. After a set number of correct choices, the correct answer (i.e. the rule governing which stimulus is correct) is changed by the computer, requiring the individual to learn from feedback and detect the new rule. The target variable for this analysis was the total number of errors made during the task, adjusted for the level of difficulty that the subject was able to reach.

Stop Signal Task (SST): The SST assesses facets of motor inhibition, which is reflective of motor impulsivity. During the task, the computer displays sequences of arrows that face either left or right. The subject is asked to press one of two buttons corresponding with the left and right arrows displayed on the screen. After a training phase, audible "beeps" are introduced after certain arrows, and participants are instructed to not press a button for arrows after which there is a "beep" until the next arrow is displayed. The length of time between the arrow and sound varies over the course of the trial, depending on the participant's success in inhibiting the initial motor response. The target measure for the task is the Stop-Signal Reaction Time (SSRT); this variable is an estimate of the time taken by the individual's brain to stop a response that would normally be made. Longer SSRTs equate to worse response inhibition.

Cambridge Gambling Task (CGT): The CGT assesses risk-taking and decision making abilities in the context of a gambling task. During the task, participants are shown a series of ten boxes, with varying proportions of those colored either red or blue. A smaller yellow square is hidden underneath one of the displayed boxes, and participants are instructed that it has an equal chance of being under any given box on the screen. Participants are then asked to select either the red set of boxes or the blue set of boxes, corresponding to which colour box they believe the yellow square is underneath. After selecting, the participant chooses a point total to bet from their "point bank", corresponding to their wager that they correctly identified which colour the yellow square will appear beneath. The points are selected from another box on the screen that shows progressively increasing point values (switch to decreasing half-way through the task) from $5 \%$ to $95 \%$ of the total available points. If 
correct, the points are doubled for use in future trials; if incorrect, the participant loses the wagered points. Target variables for the measure are overall proportion bet, quality of decision making, and risk adjustment. Overall proportion bet shows the proportion of available points the participant typically chose during the course of the task. Quality of decision making reflects proportion of times the participant chose the colour box with the greatest number present on the screen, corresponding with the greatest likelihood of containing the yellow square. Risk adjustment indicates and individual's tendency to modify betting patterns based on odds of their choice being correct (e.g. betting less for 1:1 odds, and more for 4:1 odds).

Spatial Working Memory (SWM): The SWM assesses spatial working memory related to retaining and manipulating spatial information. The task includes a series of puzzles containing multiple squares. Participants are instructed that smaller blue squares have been hidden under the squares displayed one at a time, and they need to find enough to fill a bar displayed at the edge of the screen. They are then informed that after finding a blue box under a larger box once, it is not possible to find another in that location for the remainder of that particular puzzle. The target variables for this task is the total number of errors made during the task, in which the participant selects a large square with no blue square underneath, and the quality of the strategy used when solving the puzzles (lower strategy scores equate to better strategy use).

One Touch Stocking of Cambridge (OTS): The OTS assesses executive planning skills, and follows a similar procedure to the classic Tower of London task. During the paradigm, participants are asked to visualize moving balls between sets of tubes displayed on the screen to match an example shown at the top of the screen. Upon solving the puzzle mentally, they are then asked to touch the minimum number of moves they believe the puzzle will take from a list of numbers from 1-9 displayed at the bottom of the screen. The target measure for thus analysis was the number of puzzles solved on the first choice during the task.

\subsection{Statistical analysis}

Demographic, clinical, and cognitive characteristics of the PSB subjects were compared to controls using independent t-tests for continuous variables (student t-tests, or Welsh t-tests for measures with unequal variance between groups), and chi-square (or Fisher's exact test for small cell sizes) for categorical variables. All $\mathrm{p}$ values were reported two-tailed, uncorrected. Significance was defined as $\mathrm{p} \leq 05$. No correction was undertaken for multiplicity due to the exploratory nature of the study. Bonferroni correction would have been overly conservative for this exploratory analysis (see 26). With the sample size obtained for this study, the study had $\sim 80 \%$ power to detect a statistically significant difference between groups on a given variable, assuming medium effect size 0.4 , and alpha $=0.05$ (i.e. without Bonferroni correction). Had Bonferroni correction been used, the study would have had $<40 \%$ power to detect such a group difference on a given measure, resulting in an unacceptably high risk of type II error.

Effect sizes were also calculated. Effect sizes for the equality of sets of mean differences between groups are reported in terms of Cohen effect size index ("d") or based on tests of 
the equality of 2 or more distributions over a set of 2 of more categories (X2 tests) ("w"). A $\mathrm{d}$ of .2 is considered a small effect size, .5 is medium, and .8 is large; a $\mathrm{w}$ of .1 is considered small, .3 is medium, and .5 is large (Cohen, 1988).

\section{Results}

A total of $54(11 \%)$ participants reported current PSB. The analysis showed that the PSB group was significantly older $(\mathrm{p}=.005)$, reported an earlier age of both first sexual experience $(\mathrm{p}=.031)$ and alcohol use ( $\mathrm{p}<.001)$, and had a higher body mass index ( $\mathrm{p}=.001)$.

For self-report measures, the PSB group reported significantly higher scores on all three submeasures of the BIS (attention: $\mathrm{p}=.008$; motor: $\mathrm{p}=.002$; non-planning: $\mathrm{p}=.002$ ), lower overall self-esteem $(\mathrm{p}<.001)$, greater emotional dysregulation $(\mathrm{p}=0.002)$, and lower quality of life $(p<.001)$. Internal consistency for the scales were good (Cronbach's alpha 0.79 or higher).

In terms of cognitive findings, the PSB group versus controls showed worse overall spatial working memory $(\mathrm{p}=.005)$, spatial working memory strategy $(\mathrm{p}=.028)$, motor inhibition $(\mathrm{p}=$. $048)$, and executive planning ( $\mathrm{p}=.028)$. The PSB group also bet a significantly greater proportion of their total points during the CGT versus controls ( $\mathrm{p}=.008)$.

Cronbach's alphas for the main scales used in the study were as follows: Barratt alpha $=0.80$, DERS $=0.79$,

Rates of comorbidities also differed significantly between the two groups. The PSB group reported higher prevalence rates of several general psychiatric disorders, including major depressive disorder $(\mathrm{p}<.001)$, suicidality $(\mathrm{p}=.038)$, agoraphobia $(\mathrm{p}=.010)$, alcohol use disorder $(\mathrm{p}<.001)$, and antisocial personality disorder $(\mathrm{p}=.001)$. The PSB group also reported greater rates of gambling disorder $(\mathrm{p}=.018)$, and binge-eating disorder $(\mathrm{p}=.034)$, which are considered impulse control disorders.

\section{Discussion}

In the present analysis, 54 participants (11\%) reported current PSB. This prevalence is, as expected, higher than the prevalence rates reported for compulsive sexual behavior in young adults (Black et al., 1997; Derbyshire and Grant, 2015). This analysis also indicated that PSB was associated with worse quality of life, lower self-esteem, and higher rates of comorbidities across several disorders. Furthermore, the PSB group showed deficits across several neurocognitive domains, including motor inhibition, spatial working memory, and an aspect of decision making.

One notable result from this analysis is that PSB shows significant associations with a number of deleterious clinical factors, including lower self-esteem, decreased quality of life, elevated BMI, and higher comorbidity rates for several disorders. A potential explanation for this association is that PSB is the underlying problem from which these other problems extend. Previous research on similar populations has noted that features such as shame are common amongst patients struggling with sexual behaviors (Dhuffar and Griffiths, 2014; Reid et al., 2014). These findings are consistent with the present data, as it is probable that 
individuals who feel socially isolated and stigmatized may be more likely to endorse lower self-esteem and quality of life, as these features may be intertwined with interpersonal relationships. Thus, it is possible that PSB gives rise to a host of secondary problems, ranging from alcohol dependence and depression to deteriorations in quality of life and selfesteem. This characterization would indicate it may be possible to ameliorate secondary symptoms such as depression and alcohol use by addressing problems with PSB directly during treatment.

Conversely, it is also possible that PSB should instead be characterized as a coping mechanism occurring in response to the myriad other problems identified in this analysis, such as alcohol use or depression. From this perspective, rather than characterizing PSB as a core pathology which elicits additional problems, it may instead be perceived as a way to cope with persistent negative emotions and moods, such as those that may accompany depression. This characterization fits with several aspects of the present findings, particularly the greater level of emotional dysregulation identified in the PSB group. One possibility may be that individuals with poor emotional regulation are more likely to experience periods of depression, during which they struggle to manage problems with their mood. As a response to this difficulty, they may pursue alternative ways to bolster their mood, which could take the form of PSB or other behaviors, such as alcohol, another common factor amongst the PSB group. This is consistent with previous studies on disordered sexual behavior, which have shown greater sexual interest in states of depression or anxiety, with several indicating a more unique response amongst those engaging in more compulsive forms of sexual behavior (Bancroft and Vukadinovic, 2004; Grov et al., 2010; Lykins et al., 2006). From this perspective, rather than identifying any particular clinical problem as a focal point for treatment, it may be best to help patients manage problems with emotional regulation, ideally providing coping mechanisms that do not rely on activities and behaviors that have been problematic in the past, such as PSB.

While both of these possibilities offer potential explanations for the present findings using distinct directions of causality, it is also possible that the clinical features identified in the PSB group are actually the result of a tertiary variable which gives rise to both PSB and the other clinical features. One potential factor filling this role could be the neurocognitive deficits identified in the PSB group, particularly those relating to working memory, impulsivity/impulse control, and decision making. From this characterization, it is be possible to trace the problems evident in PSB and additional clinical features, such as emotional dysregulation, to particular cognitive deficits. Issues related to impulsivity may be particularly notable, as both the BIS and SSRT showed that the PSB group was significantly more impulsive that other participants. This explanation is also fitting with other findings from the analysis, such as the earlier age of first sexual behavior and alcohol use, suggesting that problems with impulsivity may be evident from an earlier age than the onset of PSB and other problems.

By isolating neurocognition as the central characteristic identifying participants with PSB, the current findings may suggest that manifestations of these neurocognitive issues give rise to the difficulties with emotional regulation reported previously, as individuals with PSB may struggle with the processes necessary to develop well-coordinated and effective coping 
mechanisms. Furthermore, these issues with impulsivity could impair the ability to mediate the motor impulse to engage in sexual behaviors, consistent with the deficits in motor inhibition seen on the SSRT. If the cognitive problems identified in this analysis are actually the core feature of PSB, this may have notable clinical implications. Rather than working to treat problems related to either PSB or comorbid problems, it may be more effective to address the underlying problems in neurocognition. In order to tailor treatment more directly to the needs of patients with PSB, clinicians may be able to develop treatment options emphasizing strategies to mediate impulsivity, and develop more consistent coping mechanisms to manage emotional dysregulation.

There were, however, several limitations to the present analysis. One issue is that the sample only included young adults. Thus, it is possible that this analysis did not capture cognitive issues and clinical associations that only manifest after a greater duration of illness. Additionally, the present study did not include a dimensional measure of severity (we are aware of no severity measure for this subsyndromal level of sexual behavior) (Reid, 2015), thus it was not possible to assess the role of neurocognition on the severity of PSB. Due to this limitation, the analysis could not determine whether these factors showed significant associations with any specific aspects of PSB or overall severity of PSB symptoms. We did not correct for multiple comparisons as the sample size was not sufficient to enable this without unacceptable loss of statistical power. Therefore, it will be important for future studies to attempt replication of these findings in a larger sample. Cell sizes for some of the categorical data were small and caution is warranted in interpretation. For example, some impulse control disorders were relatively uncommon in both groups, and hence statistical power to detect group differences would have been limited.

Although the present analysis is unable to resolve the direction of causality for these factors, it does highlight the salient problems affecting patients with PSB. These findings suggest that individuals with PSB struggle with a number of issues, including higher comorbidity rates, greater emotional dysregulation, and select neurocognitive deficits. While the majority of individuals are able to approach sexual behavior in a healthy, constructive manner, these problems suggest that for those who struggle to control these behaviors, the related problems can have a notable effect on quality of life any many other facets of wellbeing. Thus, PSB is likely an important consideration for clinicians working with young adult populations, further highlighting the importance of screening for problems with sexual behavior across many age and gender groups. Future research assessing the importance of neurocognition in treatment may be highly beneficial, as it may be possible for clinicians to implement better screening and treatment practices based on the unique neurocognitive profile evident in patients with PSB. While data on PSB remain limited, the present findings highlight the importance of expanding and clarifying our understanding of neurocognition and clinical presentation in individuals struggling with PSB.

\section{Acknowledgements}

This research was supported by a grant from the National Center for Responsible Gaming (Centers of Excellence in Gambling Research Grant). 


\section{References}

1. Agrawal A, Bucholz KK, Lynskey MT. DSM-IV alcohol abuse due to hazardous use: a less severe form of abuse? J Stud Alcohol Drugs. 2010; 71:857-863. [PubMed: 20946742]

2. Bancroft J, Vukadinovic Z. Sexual addiction, sexual compulsivity, sexual impulsivity, or what? Toward a theoretical model. J Sex Res. 2004; 41:225-234. [PubMed: 15497051]

3. Barratt ES. Anxiety and Impulsiveness Related to Psychomotor Efficiency. Percept Mot Skills. 1959; 9:191-198.

4. Black DW, Kehrberg LL, Flumerfelt DL, Schlosser SS. Characteristics of 36 Subjects Reporting Compulsive Sexual Behavior. Am J Psychiatry. 1997; 154:243-249. [PubMed: 9016275]

5. Carneiro E, Tavares H, Sanches M, Pinsky I, Caetano R, Zaleski M, Laranjeira R. Gambling onset and progression in a sample of at-risk gamblers from the general population. Psychiatry Res. 2014; 216:404-411. [PubMed: 24656520]

6. Chen CM, Dufour MC, Yi HY. Alcohol consumption among young adults ages 18-24 in the United States: Results from the 2001-2002 NESARC survey. Alcohol Res Health. 2005; 28:269-280.

7. Cohen, J. Statistical Power Analysis for the Behavioral Sciences. second ed. Academic Press; New York: 1988.

8. Courtney KE, Polich J. Binge drinking in young adults: Data, definitions, and determinants. Psychol Bull. 2009; 135:142-156. [PubMed: 19210057]

9. Derbyshire KL, Grant JE. Compulsive sexual behavior: a review of the literature. J Behav Addict. 2015; 4:37-43. [PubMed: 26014671]

10. Dhuffar MK, Griffiths MD. Understanding the role of shame and its consequences in female hypersexual behaviours: a pilot study. J Behav Addict. 2014; 3:231-237. [PubMed: 25592308]

11. Frisch MB, Cornell J, Villanueva M, Retzlaff PJ. Clinical validation of the quality of life inventory: A measure of life satisfaction for use in treatment planning and outcome assessment. Psychol Assess. 1992; 4:92-101.

12. Gratz KL, Roemer E. Multidimensional assessment of emotion regulation and dysregulation: Development, factor structure, and initial validation of the difficulties in emotion regulation scale. J Psychopathol Behav Assess. 2004; 26:41-54.

13. Grov C, Golub SA, Mustanski B, Parsons JT. Sexual compulsivity, state affect, and sexual risk behavior in a daily diary study of gay and bisexual men. Psychol Addict Behav. 2010; 24:487-497. [PubMed: 20853934]

14. Kaestle CE, Halpern CT, Miller WC, Ford CA. Young Age at First Sexual Intercourse and Sexually Transmitted Infections in Adolescents and Young Adults. Am J Epidemiol. 2004; 161:774-780.

15. Kann L, Kinchen S, Shanklin SL, Flint KH, Kawkins J, Harris WA, Lowry R, Olsen EO, McManus T, Chyen D, Whittle L, et al. Youth risk behavior surveillance-United States, 2013. Morb Mortal Wkly Rep Surveill Summ. 2014; 63:1-168.

16. Kuzma JM, Black DW. Epidemiology, prevalence, and natural history of compulsive sexual behavior. Psychiatr Clin North Am. 2008; 31:603-611. [PubMed: 18996301]

17. Lykins AD, Janssen E, Graham CA. The relationship between negative mood and sexuality in heterosexual college woman and men. J Sex Res. 2006; 43:136-143. [PubMed: 16817060]

18. Odlaug BL, Grant JE. Impulse-control disorder in a college sample: Results from the selfadministered Minnesota Impulse Disorders Interview (MIDI). Primary Care Companion to the J Clin Psychiatry. 2010; 12:d1-e5.

19. Patton JH, Stanford MS, Barratt ES. Factor structure of the Barratt impulsiveness scale. J Clin Psychol. 1995; 51:768-774. [PubMed: 8778124]

20. Reid RC, Temko J, Moghaddam JF, Fong TW. Shame, rumination, and self-compassion in men assessed for hypersexual disorder. J Psychiatry Pract. 2014; 20:260-268.

21. Reid RC. How should severity be determined for the DSM-5 proposed classification of Hypersexual Disorder? J Behav Addict. 2015; 4:221-225. [PubMed: 26690616]

22. Rosenberg, M. Society and the adolescent self-image. Princeton University Press; Princeton, NJ: 1965. 
23. Santelli JS, Brener ND, Lowry R, Bhatt A, Zabin LS. Multiple Sexual Partners Among U.S. Adolescents and Young Adults. Fam Plann Perspect. 1998; 30:271-275. [PubMed: 9859017]

24. Sheehan DV, Lecrubier Y, Sheehan KH, Amorim P, Janavas J, Weiller E, Hergueta T, Baker R, Dunbar GC. The Mini-International Neuropsychiatric Interview (M.I.N.I.): the development and validation of a structured diagnostic psychiatric interview for DSM-IV and ICD-10. J Clin Psychiatry. 1998; 59:22-33.

25. Young SE, Corley RP, Stallings MC, Rhee SH, Crowley TJ, Hewitt JK. Substance use, abuse and dependence in adolescence: prevalence, symptom profiles and correlates. Drug Alcohol Depend. 2002; 68:309-322. [PubMed: 12393225]

26. Bender R, Lange S. Adjusting for multiple testing--when and how? J Clin Epidemiol. 2001 Apr; 54(4):343-9. Review. [PubMed: 11297884] 


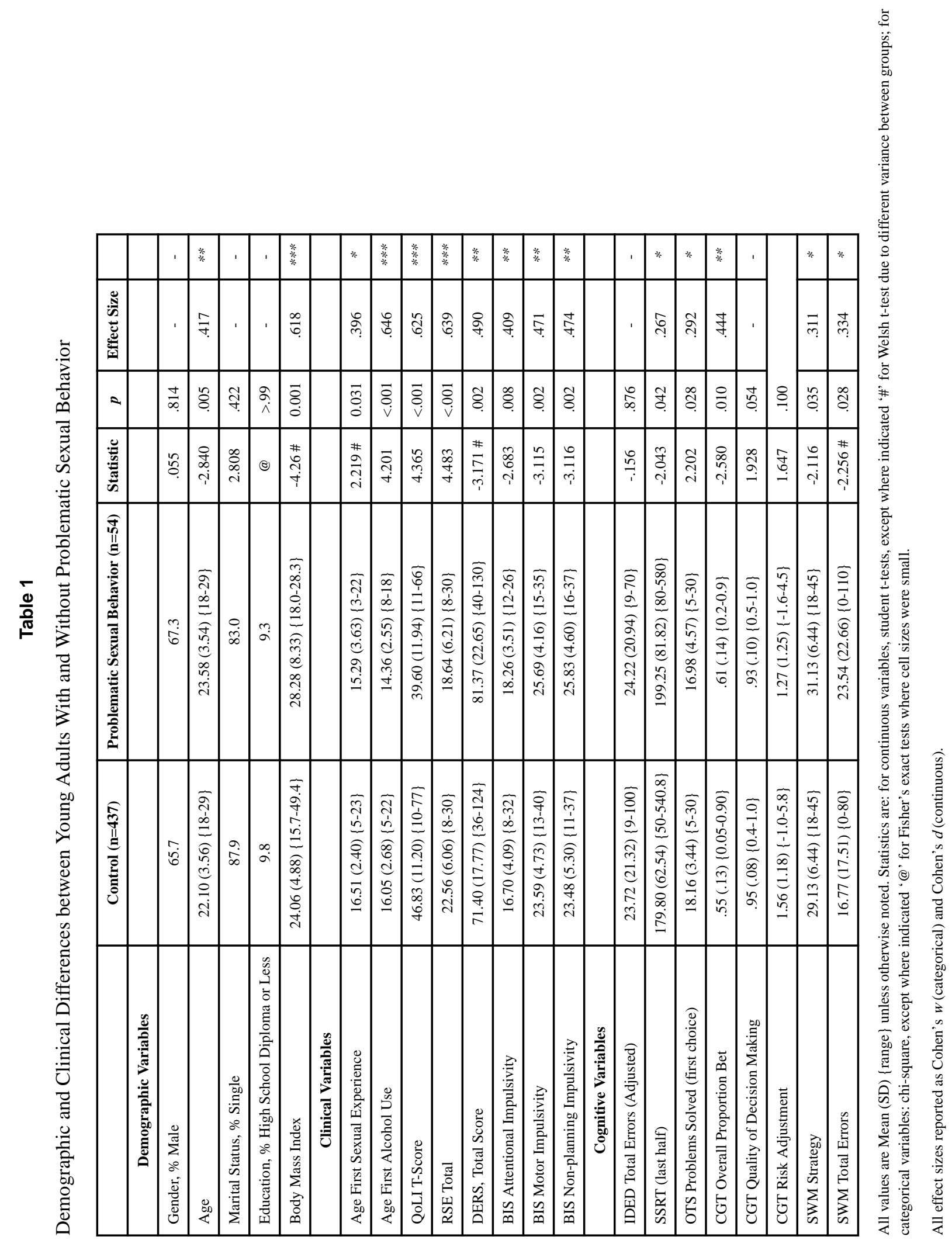

Psychiatry Res. Author manuscript; available in PMC 2017 February 28. 


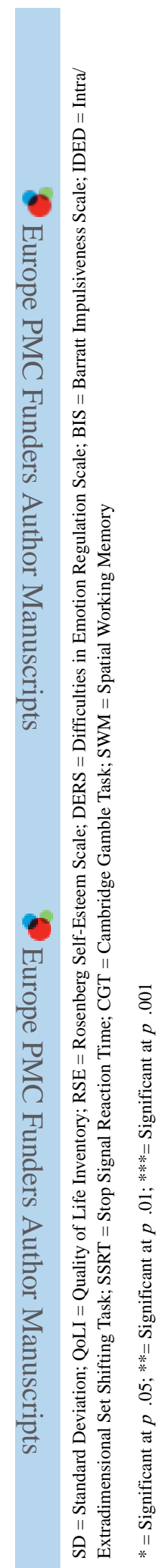

Psychiatry Res. Author manuscript; available in PMC 2017 February 28. 


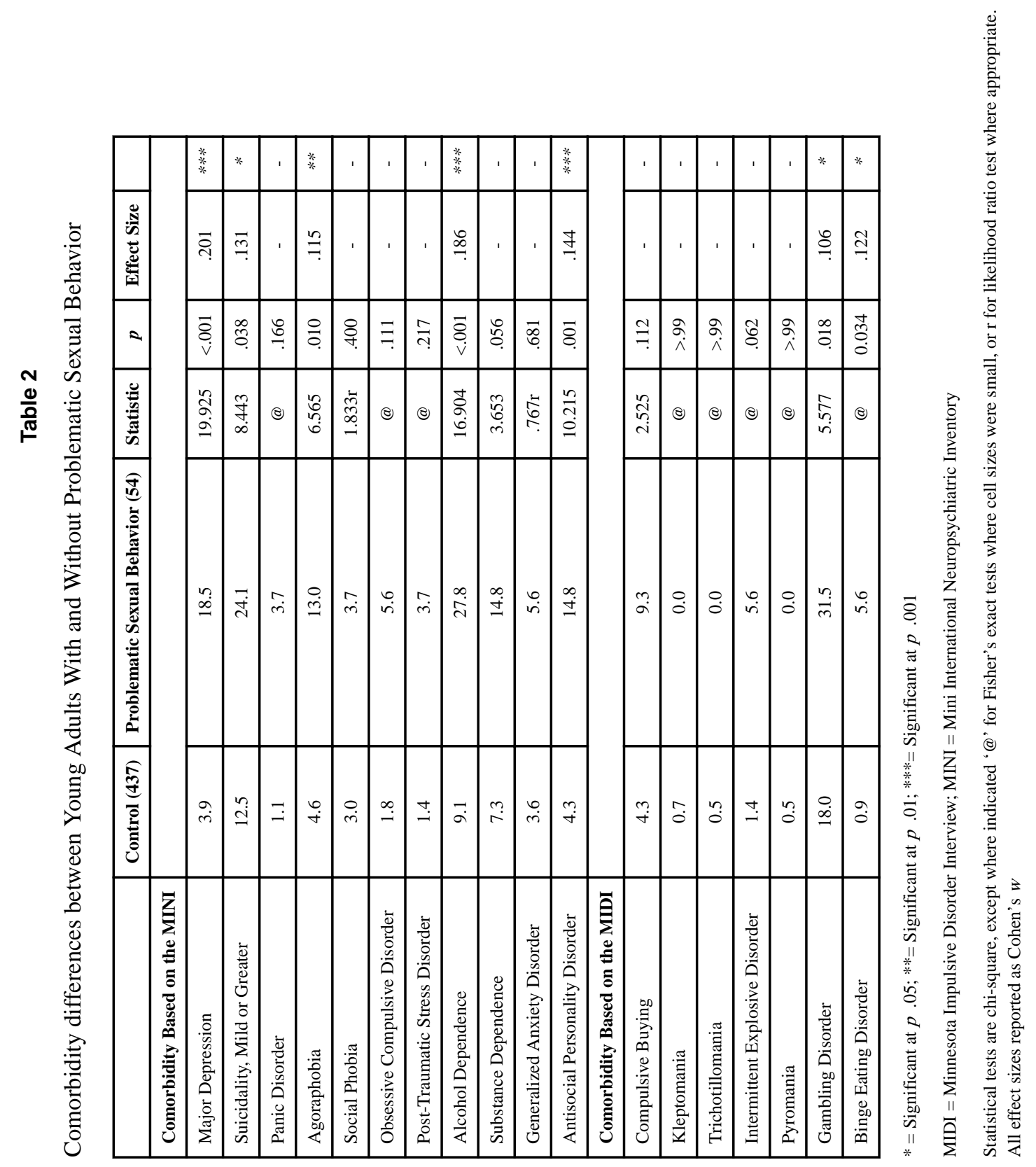

Psychiatry Res. Author manuscript; available in PMC 2017 February 28. 